\title{
Adsorption of bile salts by soya-bean flour, wheat bran, lucerne (Medicago sativa), sawdust and lignin; the effect of saponins and other plant constituents
}

\author{
By G. D. CALVERT AND ROSEMARY A. YEATES \\ Department of Clinical Biochemistry, Flinders Medical Centre, Bedford Park, \\ South Australia 5042, Australia
}

(Received 5 January 1981-Accepted 14 September 1981)

\begin{abstract}
1. The adsorption of bile salts from aqueous solution on to soya-bean flour, wheat bran, fine dry sawdust and lucerne (Medicago sativa) was measured. Adsorption on to the residuum after the first three of these substances had been extracted with organic solvents, pronase or solvents, pronase and acid (to produce lignin) was also measured.

2. The removal of saponins from soya-bean flour did not affect bile salt adsorption, and bile salts were adsorbed on to lignin and on to wheat bran (deficient in saponins). Although our methods differed from Oakenfull \& Fenwick (1978) we were unable to substantiate their conclusions that saponins are responsible for most bile salt binding to fibrous substances in vitro.
\end{abstract}

Saponins are natural products which are widely distributed in the vegetable kingdom. They are a chemically heterogeneous group of glycosides, with a polycyclic nucleus (the sapogenin moiety, with either a sterol or triterpenoid configuration) to which is attached one or more sugars (Basu \& Rastogi, 1967; Birk, 1969). Saponins are poorly absorbed from the alimentary tract, and are found in a number of preparations of fibre. It has been suggested that adsorption of bile salts to plant fibre in the gastrointestinal tract may depend on the presence of saponins bound to the fibre (Oakenfull \& Fenwick, 1978). The finding that saponins (from Saponaria officinalis) fed to rats or pigs increased faecal bile acids and neutral sterols (Oakenfull et al. 1979; Topping et al. 1980) supports this suggestion. Lucerne saponins in the diet prevent diet-induced hypercholesterolaemia in monkeys (Malinow et al. 1977). It has also been proposed that the hypocholesterolaemic effect of a textured soya-bean product (replacing animal protein in a low-cholesterol diet; Sirtori et al. 1977; Carroll et al. 1978; Sirtori et al. 1979) may be due in part to soya-bean saponins (Potter et al. 1979).

We examined the in vitro adsorption of bile salts to plant material including soya-bean flour fractions and lignin from bran or fine sawdust, with attention to the effects of removal of protein and saponins on bile salt binding. Although our experimental methods differed from those of Oakenfull \& Fenwick (1978) in several respects, we attempted to substantiate their findings that saponins appear to be responsible for most in vitro binding of bile salts to fibre, and that lignin in vitro does not bind appreciable quantities of bile salts.

MATERIALS AND METHODS

Two batches of soya-bean flour were obtained from commercial sources. One had been extracted with hexane (flour B), while the other had not (flour A). A sample of flour B was exhaustively extracted with ethanol-water $(80: 100, \mathrm{v} / \mathrm{v})$ in a commercial apparatus at room temperature with frequent stirring; this was flour $\mathrm{C}$. Before ethanol extraction flour B contained $22 \mathrm{~g}$ soya-bean saponins $/ \mathrm{kg}$; after extraction the saponin content of flour $\mathrm{C}$ was $4 \mathrm{~g} / \mathrm{kg}$ flour. Wheat bran was obtained from Purina Health Foods Company, Melbourne, Australia. Very fine powdery dry Pinus insignis sawdust was obtained from a 
local timber merchant. Dried lucerne (Medicago sativa) leaf was obtained from Bloom's Health Foods Pty Ltd, Sydney and the particles chopped with a scalpel blade so that they were less than $3 \times 1 \times 0.5 \mathrm{~mm}$ in size.

To estimate saponin content $20 \mathrm{~g}$ dried material was extracted for $16 \mathrm{~h}$ with $200 \mathrm{ml}$ hot ethanol-water $(80: 100, \mathrm{v} / \mathrm{v})$ in a Soxhlet apparatus. The extract was subjected to thin-layer chromatography on Kieselgel 60F 254 (Merck), using butanol-ethanol-aqueous ammonia solution $\left(300 \mathrm{~g} \mathrm{NH}_{3} / \mathrm{kg}\right)(35: 10: 25, \mathrm{v} / \mathrm{v})$ as the liquid phase, followed by visualization of saponin spots with sulphuric acid-ethanol $(10: 90, \mathrm{v} / \mathrm{v})$ and densitometric quantitation, with dried weighed soya-bean saponin extract prepared identically as a standard.

Phospholipids were estimated as phosphorus in the total lipid extract (Folch et al. 1957) by the method of Bartlett (1959).

\section{Bile salt binding}

Experiments on bile salt adsorption were carried out using bile salts dissolved in aqueous buffer. Phosphate buffer $(0.04 \mathrm{M}), \mathrm{pH} \mathrm{6.74}$, was made by mixing equal volumes of $0.04 \mathrm{M}$-disodium hydrogen phosphate and $0.04 \mathrm{M}$-sodium dihydrogen phosphate solutions. For some experiments the $\mathrm{pH}$ was varied by altering the proportions of the two buffers used, following the procedure of Eastwood \& Hamilton (1968), or the buffer was diluted to 0.02 M with water. For most experiments bile salt at a concentration of $1 \mathrm{mM}$ and a small amount of ${ }^{14} \mathrm{C}$-labelled bile salt as tracer (The Radiochemical Centre, Amersham, England) was added to the buffer. A portion $(6 \mathrm{ml})$ of this solution and $100 \mathrm{mg}$ of the test substance were incubated with shaking in a water bath at $37^{\circ}$ for $2 \mathrm{~h}$. The radioactivity in the filtrate was measured in a liquid-scintillation counter, using an external standard to correct for quenching. Control samples, to which no adsorbent was added, were used to correct for minor radioactivity losses on glassware and filter paper. These results, using a radioactive bile salt tracer, were confirmed in some instances by measuring bile salt adsorption with the colorimetric Pettenkoffer reaction (Boyd et al. 1966). Radioactive bile salts used included the sodium salts of taurocholic, glycocholic, chenodeoxycholic and deoxycholic acids, all shown to have at least $98 \%$ radiochemical purity by thin-layer chromatography with silica gel (using $n$-butanol-water-acetic acid $(12: 5: 3, \mathrm{v} / \mathrm{v})$ as the liquid phase for taurocholate and glycocholate, or toluene-acetic acid-water $(10: 10: 1, \mathrm{v} / \mathrm{v})$ as the liquid phase for chenodeoxycholate and deoxycholate). Non-radioactive bile salts were the purest available from Sigma Chemical Company, St Louis, Mo., USA.

\section{Modification of test substances}

In some experiments the test substances were extracted with diethyl ether, shaking overnight at room temperature to remove lipid, then digested with pronase to remove protein (Smith et al. 1974), or extracted overnight with hot ethanol-water $(80: 20, \mathrm{v} / \mathrm{v})$ in a Soxhlet apparatus.

Lignin ('Klason lignin') was produced by acid digestion after serially-extracting wheat bran and fine dry Pinus insignis sawdust with diethyl ether (overnight), pronase then a hot solution of ethanol-water $(80: 20, \mathrm{v} / \mathrm{v})$ (overnight). The procedure of Eastwood \& Hamilton (1968), also used by Oakenfull \& Fenwick (1978), was followed in detail, with the exception that these authors extracted saponins with ethanol-water $(80: 100, v / v)$. Soya-bean flour was subjected to this procedure, but insufficient residue remained for further testing.

\section{RESULTS AND DISCUSSION}

Comparison of bile salt binding using two methods, and assessment of assay conditions When the binding of sodium deoxycholate, taurocholate and glycocholate to soya-bean flour (containing $22 \mathrm{~g}$ or $4 \mathrm{~g}$ saponins/ $\mathrm{kg}$ flour), bran and lucerne was compared, the results 


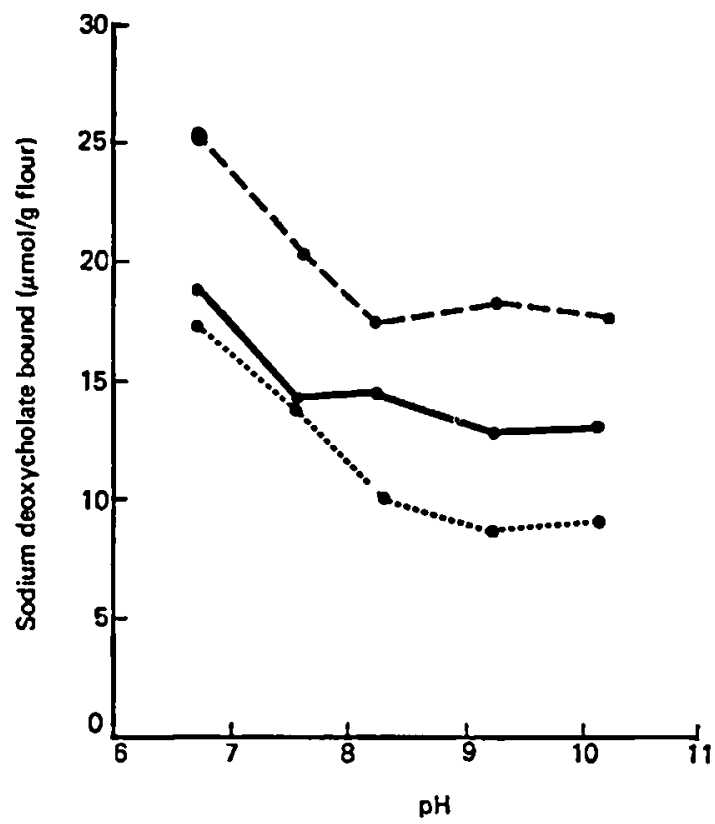

Fig. 1. The effoct of $\mathrm{pH}$ of the buffered incubation solution on the adsorption of sodium deoxycholate

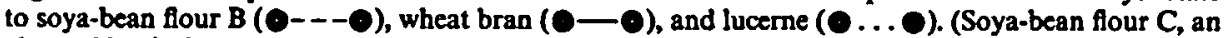
almost identical curve to that for soya-bear: flour $B$, is not shown).

with either the Pettenkoffer colorimetric method or the simpler radioactive tracer method were identical within the limits of experimental error. The within-batch coefficient of variation of deoxycholate binding using the radioactive tracer method was $3 \cdot 2$ for soya-bean flours B and C (using a standard formula to estimate variance from duplicate measurements; Brown et al. 1957). The between-batch coefficients of variation for the same sets of measurements were 6.2 (flour B) and 4.9 (flour C). The coefficient of variation of binding of other bile salts using the radioactive tracer method was very similar. We used the radioactive tracer method for quantitating bile salt binding in all other experiments. We wished to compare our results particularly with those of Eastwood \& Hamilton (1968) and Oakenfull \& Fenwick (1978), and therefore examined our assay conditions.

The ionic strength of the phosphate buffer in which the incubations were performed affected binding. In sixteen experiments, each in duplicate, the deoxycholate binding in 0.02 M-buffer to soya-bean flour (containing $22 \mathrm{~g}$ or $4 \mathrm{~g}$ saponins $/ \mathrm{kg}$ flour), bran and sawdust, with or without previous pronase digestion, was $89 \pm 2 \%$ (mean \pm SEM) of the binding in $0.04 \mathrm{M}$-buffer (the latter being the molarity used in all our other experiments). Oakenfull \& Fenwick (1978) used 0.02 M-phosphate buffer in their experiments on bile salt binding to various substances.

The effect of $\mathrm{pH}$ on deoxycholate binding to soya-bean flour, bran and lucerne was also investigated. The $\mathrm{pH}$ of the solution was checked with a $\mathrm{pH}$ meter before and after incubation. At pH 6.74 (the pH used for all other experiments), these substances bound some $7.5 \mu \mathrm{mol}$ deoxycholate $/ \mathrm{g}$ more than at $\mathrm{pH} 8.25-10.1$ (Fig. 1). These findings are similar to those reported by Eastwood \& Hamilton (1968) for bile salt binding to dry grain. Oakenfull \& Fenwick (1978) performed their incubations for similar studies at pH 6.74.

Deoxycholate binding to soya-bean flour, bran and lucerne after $2 \mathrm{~h}$ incubation at $26^{\circ}$ was identical to that after incubation at $37^{\circ}$, the temperature used in our other experiments. (Oakenfull \& Fenwick (1978) incubated fibre and bile salts in similar studies at $25^{\circ}$.) 


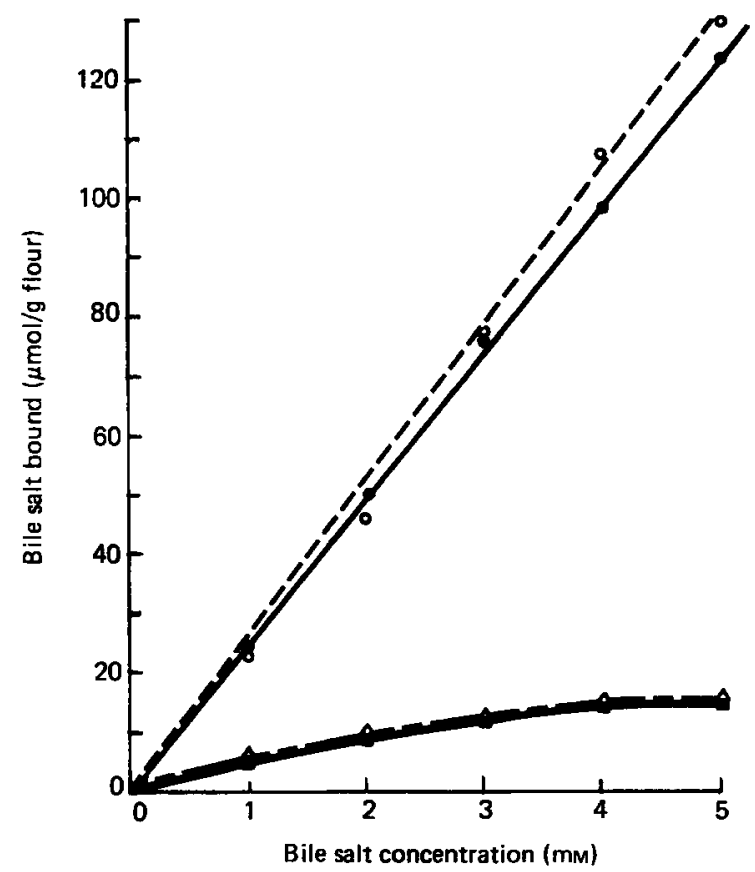

Fig. 2. The relationship between bile salt adsorption to soya-bean flour $B$, and the bile salt concentration in the incubation solution. Unconjugated bile acids (sodium deoxycholate, $\mathrm{O}_{---} \mathrm{O}$, and sodium chenodeoxycholate, - - ), bound much more avidly to the test substrate at all concentrations tested than did conjugated bile acids (sodium glycocholate, $\Delta---\Delta$, and sodium taurocholate, $\mathbf{\Delta} \mathbf{\Delta})$.

After the soya-bean flour had been soaked in water its ability to bind deoxycholate was unchanged. (A similar result was obtained by Eastwood \& Hamilton (1968)).

Both saponin-rich and saponin-depleted soya-bean flour were incubated with deoxycholate under the usual assay conditions for $0.25,0.5,1,2,3,4$ and $5 \mathrm{~h}$. Bile salt binding was maximal at $0.25 \mathrm{~h}$. Both we and Oakenfull \& Fenwick (1978) routinely incubated test substances and bile salts for $2 \mathrm{~h}$.

Comparison between binding of conjugated and unconjugated bile salts

Sodium chenodeoxycholate, deoxycholate, taurocholate and glycocholate binding at various concentrations to bran and soya-bean flour were tested. A typical experimental result is shown in Fig. 2. In all experiments, similar quantities of sodium deoxycholate and chenodeoxycholate and much smaller quantities of the conjugated bile salts, taurocholate and glycocholate were bound. Eastwood \& Hamilton (1968) also found that the conjugated bile salts bound less avidly than the unconjugated bile salts.

As we could demonstrate no statistically significant difference in binding to various bran and soya-bean flour preparations between the primary bile salt, sodium chenodeoxycholate, and the secondary bile salt, sodium deoxycholate, at $\mathrm{pH} 6.74$ and as we wished to compare our findings with those of Oakenfull \& Fenwick (1978) who used sodium cholate and deoxycholate, we have reported our findings on deoxycholate but not those on chenodeoxycholate in Table 1 and 2.

Sodium deoxycholate binding to soya-bean flour, wheat bran and sawdust Our findings mentioned previously, on the binding of bile salts to 'fibrous' substances and soya-bean flour, were similar to those of Eastwood \& Hamilton (1968).

Although our method differed from that used by Oakenfull \& Fenwick (1978), the 
differences in methodological details, examined previously, seemed to produce only minor quantitative effects in our hands. We went on to re-examine the major findings of Oakenfull \& Fenwick (1978), that bile salts did not adsorb to lignin and that their adsorption to plant material seemed to depend on the presence of saponins bound to the test substance or fibre.

Deoxycholate binding to soya-bean flour (flour which was untreated (flour A), hexaneextracted (flour B) and hexane- and ethanol-extracted (flour C)) as well as to wheat bran and very fine dry Pinus insignis sawdust was measured. Flour $\mathrm{C}$ was from the same batch of flour as flour B, but was saponin-depleted. These substances were treated with ethanol, diethyl ether, hexane and pronase, to differentiate the components which affected sodium deoxycholate binding (Table 1). It can be seen that there are considerable differences between different soya-bean-flour preparations. It is apparent, though, that saponin extraction with ethanol-water $(80: 100, \mathrm{v} / \mathrm{v}$, vide Oakenfull \& Fenwick (1978)) results in no major systematic change in deoxycholate binding (as soya-bean flour $\mathrm{C}$ differs from soya-bean flour B in having had saponins extracted with ethanol), whether or not protein is removed or other extractions performed.

Our findings in this respect differ from those of Oakenfull \& Fenwick (1978) who found that soya-bean flour bound very much less deoxycholate after saponin depletion. Our results also differ from those of Oakenfull \& Fenwick (1978) in that we obtained appreciable and consistent deoxycholate binding to sawdust and wheat bran whereas they could not demonstrate detectable binding to either of these substances.

The differences between soya-bean flours A and B were very great. Bile salt binding to soya-bean flour A is much less than binding to flour B (hexane-extracted flour from a different source). This difference remained with all treatments. We did not perform a detailed analysis of these flours, and cannot suggest a reason for the discrepancy. These values perhaps provide a caveat that flour from different sources may have quite different properties.

Part of the mass of the soya-bean flours and bran was removed by extraction with ethanol, diethyl ether or hexane, or pronase digestion, so that it was possible to estimate the deoxycholate binding for which each extracted fraction was responsible (Table 2).

Phospholipid may have accounted for some of the bile salt binding to soya-bean flour. Phospholipid made up $17 \mathrm{~g} / \mathrm{kg}$ for soya-bean flours A and B (not treated with ethanol), while phospholipid made up only $0.1 \mathrm{~g} / \mathrm{kg}$ for soya-bean flour $\mathrm{C}$ (ethanol-extracted). Lecithin adsorbed on to sawdust ( 20 or $40 \mathrm{mg} / \mathrm{g}$ sawdust) bound $46 \mu \mathrm{mol}$ deoxycholate $/ \mathrm{g}$ lecithin, i.e. lecithin made a very minor contribution to deoxycholate binding in soya-bean flour. Both phospholipid and saponins were removed by prolonged ethanol extraction in a Soxhlet apparatus, after which neither saponins nor phospholipid could be detected (by chloroform-methanol extraction (Folch et al. 1957) or further hot-ethanol extraction).

Soya-bean flours B and C, but not soya-bean flour A, bound much less deoxycholate after pronase digestion than before. The reason for this discrepancy between flours $B$ and $\mathrm{C}$, and $\mathrm{A}$, in unknown. The change in binding after pronase in flours $\mathrm{B}$ and $\mathrm{C}$ and wheat bran suggest that protein is important in binding bile salts. It is possible also that pronase treatment removed some pectic materials, which may also play a part in bile salt binding. Neither treatment with diethyl ether, pronase, nor ethanol had any effect on the binding ability of dry Pinus insignis sawdust. Untreated lucerne which contains saponins bound deoxycholate, but no more avidly (on a weight basis) than wheat bran which contains no detectable saponin or soya-bean flour B or C.

We concluded that a number of plant constituents probably including saponins (Table 2) appeared to bind deoxycholate in vitro. Again, our findings differ from those of Oakenfull \& Fenwick (1978) who found that the removal of saponins virtually abolished deoxycholate binding to a number of substances including soya-bean flour. 


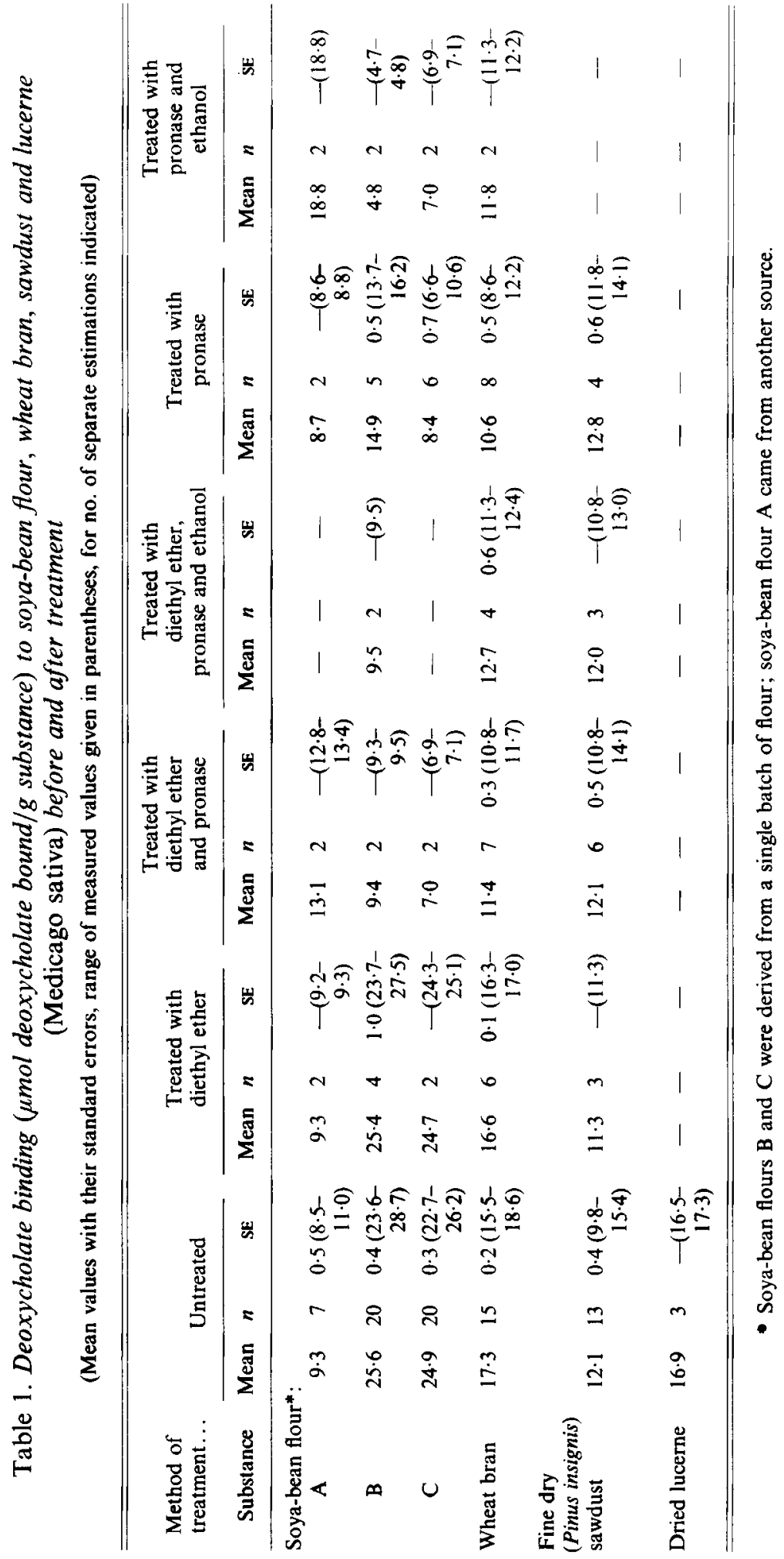


Table 2. Deoxycholate binding to components of soya-bean flour and wheat bran after various treatments (umol deoxycholate binding/g original flour or bran removed by treatment)

(Results are the means of two experiments (each in duplicate) in which sequential treatment by diethyl ether, pronase and ethanol allowed calculation by subtraction of the deoxycholate bound)

\begin{tabular}{lccc}
\hline & $\begin{array}{c}\text { Soya-bean } \\
\text { flour B* }\end{array}$ & $\begin{array}{c}\text { Soya-bean } \\
\text { flour C* }\end{array}$ & Wheat bran \\
\hline Removed by diethyl ether & $3 \cdot 5$ & 0 & 0 \\
Removed by pronase & $21 \cdot 3$ & $22 \cdot 6$ & 6.2 \\
Removed by ethanol & $5 \cdot 1$ & 0 & 0 \\
Residuum & $1 \cdot 5$ & 3.4 & 10.8 \\
\hline
\end{tabular}

- Both soya-bean flours B and C have been extracted with hexane. Soya-bean flour C was phospholipid- and saponin-depleted after subsequent ethanol extraction.

Deoxycholate binding to lignin derived from bran or sawdust

After serial treatment with diethyl ether, pronase and ethanol, soya-bean flour, wheat bran and fine dry Pinus insignis sawdust were treated with sulphuric acid to produce lignin.

After acid treatment $26 \%$ of sawdust was recovered, and $7 \%$ of bran, but there was insufficient soya-bean residue to test. The sawdust residue (lignin) bound $51 \mu \mathrm{mol}$ deoxycholate/g residue (duplicate samples), and the 'lignin' derived from wheat bran $45 \mu \mathrm{mol}$ deoxycholate/g. These findings were repeated in a second experiment.

We concluded that lignin produced from either wheat bran or fine dry Pinus insignis sawdust bound sodium deoxycholate at least as avidly as other substances tested. Our method of lignin production, by treating material with sulphuric acid after saponins had been extracted, was identical to that of Eastwood \& Hamilton (1968) and Oakenfull \& Fenwick (1978), with the exception that we extracted with ethanol-water $(80: 20, \mathrm{v} / \mathrm{v})$ rather than ethanol-water $(80: 100, \mathrm{v} / \mathrm{v})$.

These results are in substantial agreement (although we used a different bile salt) with those of Eastwood \& Hamilton (1968) who found that oak chips and sawdust bound approximately $10 \mu \mathrm{mol}$ sodium cholate $/ \mathrm{g}$, and lignin produced from wood wool, sawdust or dry grain bound approximately $11 \mu \mathrm{mol}$ sodium cholate/g. Balmer \& Zilversmit (1974) also demonstrated the in vitro binding of a bile salt to lignin. In their experiments sodium taurocholate bound to lignin more avidly than to soya-bean meal, ground wheat, maize or oats, or wheat middlings. Our results are at variance with those of Oakenfull \& Fenwick (1978) who found that very little or no sodium cholate or deoxycholate bound to sawdust or wheat bran, or lignin derived from sawdust or wheat bran. In our experiments deoxycholate bound to all these substances.

Oakenfull \& Fenwick (1978) reported that from 2.5 to $2.7 \mu \mathrm{mol}$ deoxycholate were bound/g saponin-deficient soya-bean flour which had been extracted with ethanol-water $(80: 100, \mathrm{v} / \mathrm{v})$ in a Soxhlet apparatus (cf. 18.5-18.9 $\mu \mathrm{mol}$ bound $/ \mathrm{g}$ of non-saponin-deficient soya-bean flour). Using a similar extraction procedure (soya-bean flour $\mathrm{C}$ was extracted in Dr Oakenfull's institution with ethanol-water $(80: 100, \mathrm{v} / \mathrm{v})$ and the saponin content checked in his laboratory), but using a different method of assessing bile salt binding, we found that from $22 \cdot 7$ to $26 \cdot 2 \mu \mathrm{mol}$ deoxycholate were bound/g saponin-deficient soya-bean flour (Table 1). This was not significantly different from the amount of deoxycholate bound/g of non-saponin-deficient soya-bean flour from the same batch (flour $\mathbf{B}$, binding $23 \cdot 6-28 \cdot 7 \mu \mathrm{mol}$ deoxycholate $/ \mathrm{g}$ flour). 


\section{Are these in vitro studies relevant to the in vivo action of fibre?}

Bran and chopped lucerne in our experiments bound similar amounts of deoxycholate $(17.3 \pm 0.9 \mu \mathrm{mol}$ and $16.9 \pm 0.4 \mu \mathrm{mol} / \mathrm{g}$ respectively), but bran in the diet is quite ineffective as a hypocholesterolaemic agent (Truswell \& Kay, 1976) whereas lucerne, under some circumstances, is highly effective (Naito et al. 1978). Indeed, in a recent well-controlled study the addition of wheat bran to the diet caused an increase in serum cholesterol (StrasseWolthuis et al. 1979). These clinical trials suggest that the ability of substances to bind bile salts in vitro bears little or no relationship to any hypocholesterolaemic effect observed when those substances form part of the diet. Jenkins (1979), in a recent review of the literature, has reached a similar conclusion based on different evidence, that binding or sequestration of bile salts to increase faecal loss cannot be the full explanation for the hypocholesterolaemic properties of certain dietary fibres.

In our view the discrepancy between our findings and the quite different findings of Oakenfull \& Fenwick (1978) should be resolved before any deductions on the interaction of ingested fibre and bile salt metabolism can be made. It is true that we have used different experimental methods. Nevertheless, we have not been able to produce any evidence to suggest that adsorption of bile salts to fibre depends on the presence of saponins.

This work was supported by grants from the National Heart Foundation of Australia and the National Health and Medical Research Council. We thank Dr D. G. Oakenfull and his staff, CSIRO Division of Food Research, NSW 2113, Australia, for the supply of soya-bean flours $B$ and $C$, for the extraction of saponins from flour $C$, and for the estimation of saponins in flours $B$ and $C$. Thanks are also due to Joanna Fenton and Julie-Anne Burton for secretarial assistance.

\section{REFERENCES}

Balmer, J. \& Zilversmit, D. B. (1974). J. Nutr. 104, 1319.

Bartlett, G. R. (1959). J. biol. Chem. 234, 466.

Basu, N. \& Rastogi, R. P. (1967). Phytochemistry 6, 1249.

Birk, Y. (1969). In Toxic Constituents of Plant Foodstuffs, pp. 169-210 [I. E. Liener, editor]. New York: Academic Press.

Boyd, G. S., Eastwood, M. A. \& Maclean, N. (1966). J. Lipid Res. 7, 83.

Brown, J. B., Bulbrook, R. D. \& Greenwood, F. C. (1957). J. Endocr. 16, 41.

Carroll, K. K., Giovannetti, P. M., Huff, M. W., Moase, O., Roberts, D. C. K. \& Wolfe, B. M. (1978). Am. J. clin. Nutr. 31, 1312.

Eastwood, M. A. \& Hamilton, D. (1968). Biochim. biophys. Acta 152, 165.

Folch, J., Lees, M. \& Sloane-Stanley, G. H. (1957). J. biol. Chem. 226, 497.

Jenkins, D. J. A. (1979). Lancet ii, 1287.

Malinow, M. R., McLaughlin, P., Kohler, G. O. \& Livingston, A. L. (1977). Steroids 29, 105.

Naito, H. K., Lewis, L. A. \& McNulty, W. P. (1978). Atherosclerosis 30, 27.

Oakenfull, D. G. \& Fenwick, D. E. (1978). Br. J. Nutr. 40. 299.

Oakenfull. D. G., Fenwick, D. E., Hood, R. L., Topping, D. L., Illman, R. J. \& Storer, G. B. (1979). Br. J. Nutr. 42, 209.

Potter, J. D., Topping, D. L. \& Oakenfull, D. G. (1979). Lancet i. 223.

Sirtori, C. R., Agradi, E., Conti, F., Mantero, O. \& Gatti. E. (1977). Lancet i, 275.

Sirtori, C. R., Gatti, E., Mantero, O., Conti, F., Agradi. E., Tremoli, E., Sirtori, M., Fraterrigo, L., Tavazzi, L. \& Kritchevsky, D. (1979). Am. J. clin. Nutr. 32, 1645.

Smith, M. B., Reynolds, T. M., Buckingham, C. P. \& Back, J. F. (1974). Aust. J. biol. Sci. $27,349$.

Strasse-Wolthuis, M., Katan, M. B., Hermus, R. J. J. \& Hautvast, J. G. A. J. (1979). Atherosclerosis $34,87$.

Topping, D. L., Storer, G. B., Calvert, G. D., Illman, R. J., Oakenfull, D. G. \& Weller, R. A. (1980). Am.J. clin Nutr. 33, 783.

Truswell, A. S. \& Kay, R. M. (1976). Lancet i, 367. 